\title{
An Experimental Study on the Measurement of Objective Sound under Contamination of Wind Noise
}

\author{
Y. Takakuwa ${ }^{(1)}$, M. Ohta ${ }^{(2)}$, M. Nishimura ${ }^{(1)}$ and H. Minamihara ${ }^{(3)}$ \\ (a) Faculty of Engineering, Hiroshima-Denki Inst. of Technology, 6-20-1 Nakano, Aki-ku, Hiroshima, 739-03 Japan \\ (2) Faculty of Engineering, Kinki University, 1 Umenobe, Takaya, Higashi-hiroshima, 729-17 Japan \\ (3) Faculty of Engineering, Okayama University of Science, 1-1 Ridai-chyo, Okayama, 700 Japan
}

\begin{abstract}
This paper proposes a new simple method of determining the steady sound pressure level of machinery contaminated with wind noise in the measurement of low-frequency environmental noise in windy conditions. First, the cross-correlation characteristics between the spectrum levels and the band pressure levels measured in a particular frequency range of the wind noise are discussed. Next, a simple and practical static estimation method for the objective sound is discussed by employing only the conditional mean of the spectrum. Finally, the effectiveness of the proposed method is shown through experiments made in breezy conditions in the open air. The estimation accuracy of the method is discussed and compared with that of another method employing the wind speed observed simultaneously in the vicinity of the measurement microphone.
\end{abstract}

\section{INTRODUCTION}

Wind-induced noise or wind noise is an inevitable disturbance that appears in outdoor measurements of low-frequency acoustic noises in windy conditions. It is difficult to remove the low-frequency components of the wind noise by using windscreens designed for sound level meters ${ }^{1-3}$. This is the reason why outdoor measurements of low-frequency noise are still recommended to be carried out in windless conditions in a recent proposal ${ }^{4}$. Nonetheless, the necessity of measuring the outdoor low-frequency noise from some noise sources, such as sieving machines, hot-air dryers or discharges of a dom, arises frequently even under windy conditions. Let us call the acoustic noise or the sound to be measured "the objective sound". In such cases, some other counter-measures against the wind noise are needed in order to undertake accurate measurements.

The method of making measurements with a microphone near the ground surface ${ }^{5}$ is sometimes effective, but it is not always an essential counter-measure. The synchronized integration method ${ }^{6}$ is useful when the objective sound is made artificially intermittent in steady wind noise. The method using a coherent detector ${ }^{7,8}$ is also effective but the objective sound is required to be a pure tone. The methods based on correlation techniques using multiple microphones 2,9,10 are effective but their measurement systems become somewhat large. Furthermore, dynamic estimation methods based on using a Bayesian type digital filters ${ }^{\mathbf{8}, 1,12}$ are effective for the evaluation of arbitrary probability distributions of the objective sound, but their algorithms are fairly complicated for practical use.

We have already reported two simple estimation methods of static and dynamic types in the time domain and another simple estimation method in the frequency domain ${ }^{13-16}$. In this study, in order to find more simple and practical estimation methods, first we discuss further characteristics of the wind noise. Next, we propose a new static estimation method for the objective sound contaminated by the wind noise. Finally, some of the experimental results and the effectiveness of the proposed method are shown by comparing the results with another estimation method ${ }^{16}$.

\section{WIND NOISE}

The wind noise is composed of two components ${ }^{3,17}$. The first component is the "pressure turbulence" generated by the microphone or the added windscreen located in the airstream of the wind. The spectrum of this component usually occupies the higher part of the spectral contents of the wind noise and it is dependent on the wind speed ${ }^{18,19}$. The second component is windborne turbulence in the vicinity of the microphone. The spectrum of this component is considered to be dominated by low frequency components from the experimental results of wind turbulence measurements ${ }^{20}$. Part of this component under windy conditions is also considered to be dependent on the wind speed, because much of the turbulence is originally generated by large structures or natural objects in the wind stream and is carried with the wind, although it decays with time. Thus, the wind noise, consisting of these two components described, is expected to be correlated considerably with the wind speed. This conclusion is also supported by some experimental evidence ${ }^{13-16,17}$. Here, we intend to clarify the spectrum characteristics.

\section{Spectrum characteristics}

We use the signal of the pre-amplifier output of a microphone in the frequency domain as the observed result of the wind noise. First, we notice the fact that a wind noise composed of the above pressure turbulence has a wide bandwidth and each spectrum level (SL) of the frequency components is correlated with the sound pressure level (SPL) of the wind noise. We have verified the correlation relationship with the octave band pressure levels (BPL) of the wind noise ${ }^{15}$. Here, we notice the power spectrum shapes of the wind noise and focus on the correlation relationship between 